\title{
AVALIAÇÃO DE CATALISADOR A BASE DE CONCHAS DE OSTRAS PARA A PRODUÇÃO DE BIODIESEL UTILIZANDO PLANEJAMENTO FATORIAL
}

\author{
C. D. Paula ${ }^{1}$,F. J. S. Barros ${ }^{1}$, L. M. Correia $^{1}$ e R. S. Vieira ${ }^{1 *}$. \\ ${ }^{1}$ Universidade Federal do Ceará, Depto de Eng. de Química, Grupo de Pesquisas em Separação por Adsorção \\ rodrigogpsa@gmail.com.br
}

Submetido 10/10/2016 - Aceito 07/03/2017

DOI: $10.15628 /$ holos.2017.5204

\section{RESUMO}

O Biodiesel é um combustível renovável derivado de óleos vegetais ou de gordura animal que pode substituir total ou parcialmente o óleo diesel derivado de petróleo. Possui algumas vantagens quando comparado ao diesel fóssil tais quais biodegrabilidade e não toxicidade. A transesterificação com álcool de cadeia curta e catalisadores básicos é a forma mais usual de produção desse combustível. Otimizar a proporção de catalisador e álcool empregados na reação é importante do ponto de vista técnico e econômico, possibilitando identificar as quantidades mínimas necessárias para obter um produto final com alto teor de éster. O objetivo desse trabalho foi avaliar o processo de produção de biodiesel, realizando um estudo das variáveis de processo que influenciam na reação de transesterificação do óleo de girassol utilizando um catalisador heterogêneo básico obtido da farinha das conchas de ostras, de forma a se obter as condições operacionais que levam a uma maior conversão em biodiesel. As reações de transesterificação do óleo de girassol com metanol foram realizadas em um reator batelada a uma temperatura de $65{ }^{\circ} \mathrm{C}$ por 5 horas. Os experimentos foram realizados de acordo com o planejamento experimental $3^{2}$ acrescidos de três pontos centrais, resultando em 12 experimentos. As variáveis estudadas foram: concentração do catalisador $(1 \%, 2 \%$, $3 \%)$, razão molar óleo: metanol (1:06, 1:09, 1:12). As condições otimizadas da reação foram $X_{R M}(1: 12$ mol:mol), $X_{\text {CAT }}(2 \%$ wt.\%) com produção de $99,99 \%$ ( \pm $0,04 \mathrm{wt}$ ) de biodiesel metílico de girassol. No presente trabalho o parâmetro de avaliação foi a conversão em biodiesel, determinada por cromatografia gasosa.

PALAVRAS-CHAVE: Biodiesel, Ostras, Planejamento Experimental.

\section{EVALUATION OF OYSTERS BASED CATALYST FOR BIODIESEL PRODUCTION USING EXPERIMENTAL PLANNING}

\begin{abstract}
Biodiesel is a renewable fuel derived from vegetable oils or animal fat that can replace entirely or partially the diesel derived from oil. It has some advantages compared to fossil diesel, such as biodegradability and non-toxicity. The transesterification reaction with a short chain alcohol and basic catalyst is the most usual form of this fuel production. Optimize the proportion ratio of catalyst and alcohol used in the reaction is important from a technical and economic point of view, making it possible to identify the minimum amounts necessary to obtain a final product with high ester content. The aim of this study was to evaluate the biodiesel production process, conducting a study of the process variables that influence the transesterification of sunflower oil using a basic heterogeneous catalyst
\end{abstract} the conditions operations leading to a higher conversion to biodiesel. The transesterification reactions of sunflower oil with methanol were performed in a batch reactor at a temperature of $65^{\circ} \mathrm{C}$ for 5 hours. The experiments were performed according to the experimental planning $3^{2}$ with additional three central points, resulting in 12 experiments. The variables studied were: catalyst concentration $(1 \%, 2 \%, 3 \%)$, molar ratio of oil: methanol (1:06, 1:09, 1:12). The optimized conditions of reaction were $X_{R M}(1: 12$ mol: $\mathrm{mol}), \mathrm{X}_{\text {CAT }}(2 \% \mathrm{wt} \%$.) with production of $99.99 \%( \pm 0.04$ wt) methyl sunflower biodiesel. In the present work, the evaluation parameter was the conversion into biodiesel determined by gas chromatography.

KEYWORDS: Biodiesel, Transesterification, Experimental Planning. 


\section{INTRODUÇÃO}

O esgotamento das fontes de energia não renováveis, como petróleo e o carvão, tem impulsionado novas pesquisas por combustíveis derivados de fontes renováveis de energia. Dentre eles destaca-se o biodiesel, que pode ser definido como uma mistura de ésteres monoalquílicos de cadeia longa, derivados de matérias primas como óleos vegetais e gorduras animais (Demirbas, 2009). O seu uso apresenta algumas vantagens, como o fato de ser um combustível não fóssil, podendo substituir total ou parcialmente o diesel de petróleo em motores de ciclo diesel, sem a necessidade de qualquer adaptação (Pinto et al., 2005). O processo mais comumente usado para a produção de biodiesel é a transesterificação, que consiste na reação de um óleo vegetal ou gordura animal com um álcool na presença de um catalisador, formando glicerol e ésteres (Meher,2006). Na reação de transesterificação pode-se fazer uso da catálise homogênea ou heterogênea. Embora a catálise homogênea produza conversões elevadas em tempos relativamente curtos, a sua utilização na reação de transesterificação com óleos vegetais resulta na formação de sabão, que é uma reação paralela indesejável (Al-Widyan \& Al-Shyoukh, 2002). Além disso, os catalisadores homogêneos não podem ser reutilizados e o seu processo de separação são mais complicados, elevando os custos (Pinto et al., 2005). A produção de biodiesel utilizando catalisadores heterogêneos apresenta diversas vantagens, como a redução dos custos ao permitir a reutilização do catalisador, além de eliminar as etapas de lavagens presente na catálise homogênea (Gomes, 2008).

Durante o processo de produção do biodiesel, a transesterificação é uma reação reversível, dessa forma, convém utilizar excesso de álcool, para aumentar o rendimento mássico da reação, evitando que ocorra a formação de glicerina e interferentes, que venham a prejudicar a qualidade do combustível (Domimgos, 2008). Além da razão álcool/óleo a reação é influenciada por: tipo de álcool, tipo de catalisador, teor de catalisador, temperatura de reação, pressão, tempo de reação e pureza de reagentes (Lôbo et al., 2009). A otimização desses fatores é importante durante o processo de produção, a fim de maximizar o rendimento de biodiesel, minimizando os custos operacionais de produção. O processo de otimização, aliado ao planejamento experimental e metodologia de superfície de resposta é utilizado com finalidade de determinar as condições favoráveis de operação, visando uma maior conversão de ésteres de forma a favorecer uma elevada taxa em rendimento mássico da reação (Antolí et al., 2002; Oliveira et al., 2010).

O objetivo desse trabaho é a otimização do processo de produção de biodiesel metílico utilizando catalisador obtido a partir de farinha de conchas de ostra, variando a razão molar óleo:metanol e quantidade de catalisador através de planejamento de Plackett Burman e metodologia de superfície de resposta.

\section{MATERIAS E MÉTODOS}

\subsection{Reagentes e Materias}

O material utilizado para a reação de transesterificação foi o óleo de girassol refinado da marca Liza, adquirido em um supermercado local e metanol $(99,95 \%)$ adquirido da marca Vetec, as ostras residuais foram coletadas de restaurante local, padrão de heptadecanoato de metila com grau de pureza de 99,5 \% p/p (Marca SIGMA, EUA) e gases $\mathrm{He}, \mathrm{H}_{2}$ e Ar de grau analítico 
(WHITE MARTINS). As propriedades físicas e químicas do óleo de girassol refinado comercial são: índice de acidez $\left(0,21 \pm 0,02 \mathrm{mg} \mathrm{KOH} \mathrm{g}^{-1}\right)$, massa específica a $20{ }^{\circ} \mathrm{C}(918,80 \pm 1,30)$, viscosidade cinemática a $40{ }^{\circ} \mathrm{C}(33,53 \pm 0,34)$, os ácidos graxos encontrados correspondem ao C12:0 $(0,05 \pm$ 0,01), C14:0 (0,09 $\pm 0,01), C 16: 0(5,28 \pm 0,02), C 16: 1(0,26 \pm 0,01), C 18: 1(64,06 \pm 0,03), C 18: 2$ $(17,69 \pm 0,98), C 18: 3(9,03 \pm 0,05), C 20: 0(0,73 \pm 0,02), C 22: 0(0,37 \pm 0,01), C 22: 1(0,19 \pm 0,02)$, os ácidos graxos saturados e insaturados correspondem respectivamente, 8,89 e $91,11 \% \mathrm{p} / \mathrm{p}$. A massa molar do óleo vegetal de girassol refinado comercial foi calculado conforme descrito por Alcântara et al, 2000. A massa molar encontrada foi de $883,27 \mathrm{~g} \cdot \mathrm{mol}^{-1}$. As análises foram feitas em triplicatas e estão apresentadas com o desvio padrão (\%).

\subsection{Preparo e Ativação do Catalisador}

As conchas das ostras foram lavadas com água destilada $(5 x)$ para a remoção da matéria orgânica e moídas em moinho de bolas até obtenção de um pó fino, após esta etapa foram peneiradas e em seguida secas em estufa por 12 horas a $120^{\circ} \mathrm{C}$. O catalisador heterogêneo foi obtido a partir do tratamento térmico do precursor catalítico (farinha das conchas das ostras natural) visando a obtenção do óxido de cálcio, sendo utilizado para isso aquecimento a $900{ }^{\circ} \mathrm{C}$ em forno mufla durante 3 horas. Após a calcinação, o material foi pesado e imediatamente utilizado nas reações de transesterificação com óleo de girassol refinado e metanol. Os métodos de caracterização do catalisador foram utilizados com o objetivo de avaliar as propriedades físicas e químicas do material através das técnicas de fluorescência de raios-X (FRX), difração de raios-X (DRX) e análise termogravimétrica (TG/DTG).

\subsection{Planejamento fatorial para produção de biodiesel}

A fim de se avaliar as variáveis que influenciam no processo de produção de biodiesel foi utilizado um planejamento fatorial apresentado na Tabela 1. A matriz do planejamento experimental foi o fatorial $3^{2}$ com o ponto central em triplicata, resultando em 12 experimentos. Utilizaram-se como variáveis independentes a razão molar $\left(X_{M R}\right)$ e a quantidade de catalisador $\left(\mathrm{X}_{\mathrm{CAT}}\right)$ e como variável dependente e resposta o teor dos ésteres metílicos ( $\mathrm{Y}_{\text {FAME }}$ ).

Tabela 1 : Matriz do planejamento fatorial da reação de transesterificação.

\begin{tabular}{l|l|lcl}
\hline Variáveis & Símbolos e Unidades & \multicolumn{3}{|c}{ Variáveis Codificadas } \\
\cline { 2 - 4 } & & $\mathbf{- 1}$ & $\mathbf{0}$ & $\mathbf{1}$ \\
\hline Razão Molar & $\mathrm{X}_{\mathrm{RM}}(\mathrm{mol}: \mathrm{mol})$ & $01: 06$ & $01: 09$ & $01: 12$ \\
$\begin{array}{l}\text { óleo:metanol } \\
\text { Quantidadede Calisador }\end{array}$ & $\mathrm{X}_{\mathrm{CAT}}(\mathrm{wt} . \%)$ & 1 & 2 & 3 \\
\hline
\end{tabular}

\subsection{Reação de Transesterificação}

A reação de transesterificação foi realizada em um balão de fundo redondo com três entradas, equipado com condensador e agitador magnético. Inicialmente, no balão foram introduzidos $30 \mathrm{~mL}$ de óleo de girassol e o metanol, e posteriormente, o catalisador. A relação massa do óleo pesada, massa de catalisador e razão molar estão descritas no planejamento fatorial (Tabela 1). Após o término da reação de transesterificação, o catalisador foi separado por filtração a vácuo, e a mistura reacional foi colocada em funil de decantação para a separação das fases (ésteres metílicos e glicerina). Em seguida, o metanol em excesso foi removido da fase éster utilizando um destilador Kugelrohr. A conversão do óleo de girassol em ésteres metílicos foi 
determinada por cromatografia gasosa (CG/FID), conforme descrito na norma padrão adotada pela ANP (EN 14103).

\section{RESULTADOS E DISCUSSÕES}

A Figura 1 mostra o difratogramas de raios $X$ para o resíduo da farinha da concha da ostra natural (a) e calcinada (b). No difratograma do resíduo da farinha da concha da ostra natural foram indentificados picos característicos de calcita $\left(\mathrm{CaCO}_{3}\right)$, em $2 \theta=23,26,29,31,36,39,43$, $46,47,48,57,60,63$ e 64, possuindo estrutura romboédrica (01-085-1108 código de referência do cartão ICSD). O DRX do resíduo da concha da ostra calcinada a $900^{\circ} \mathrm{C} / 3$ horas, apresenta picos correspondentes ao $\mathrm{CaO}(18,28,29,32,34,40,47,50,54,62,64$ e 67), em acordância com dados do padrão de DRX do $\mathrm{CaO}$, apresentando estrutura cristalina do tipo cúbica (código 00044-1481 referência de cartão ICSD). Esses resultados revelam que a temperatura utilizada no processo térmico foi efeciente na mudança de fases do catalisador.

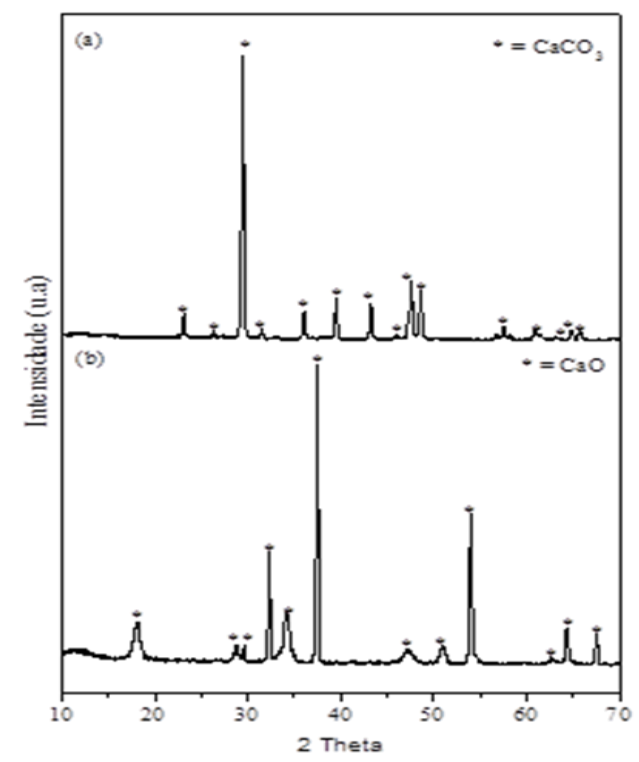

Figura 1: DRX (a) ostra natural e (b) ostra calcinada a $900 \stackrel{\circ}{\circ} / 3 \mathrm{~h}$.

As curvas TG/DTG para o resíduo da farinha das conchas das ostras naturais são mostradas na Figura 2, observa-se que a decomposição ocorre em dois eventos, com diferenças nas perdas de massa em cada um. No primeiro evento observa-se a perda de massa de $4 \%$ entre $0-210{ }^{\circ} \mathrm{C}$, que é atribuída à perda de água fisicamente adsorvida na superfície do material, de ligações de hidrogênio. $\mathrm{O}$ segundo evento, entre $550-750{ }^{\circ} \mathrm{C}$, é atribuído à perda de massa $(43,95 \%)$, está associado com a decomposição do carbonato de cálcio $\left(\mathrm{CaCO}_{3}\right)$ e a formação de óxido de cálcio (CaO). Os resultados encontrados são compatíveis com os observados por Silva (2007) e Buasri et al. (2014), que submeteram as conchas das ostras ao mesmo tratamento térmico.

Na análise de FRX foi possível obter resultados importantes dos materiais nas formas natural e calcinada. O cálcio $(\mathrm{Ca})$ é o elemento mais abundante no resíduo da ostra natural e calcinada respectivamente, $95,00 \% \mathrm{p} / \mathrm{p} \mathrm{Ca}-\mathrm{CaCO}_{3}$ na forma de calcita e 96,00 \% p/p Ca-CaO como óxido de cálcio. Outros elementos foram encontrados em menores quantidades como $\mathrm{Na}$, $\mathrm{Fe}, \mathrm{Si}, \mathrm{S}, \mathrm{Sr}, \mathrm{K}, \mathrm{Ag} \mathrm{e} \mathrm{Cl}$, considerados impurezas dos próprios materiais, visto que os mesmos tem origem em águas salinas. 


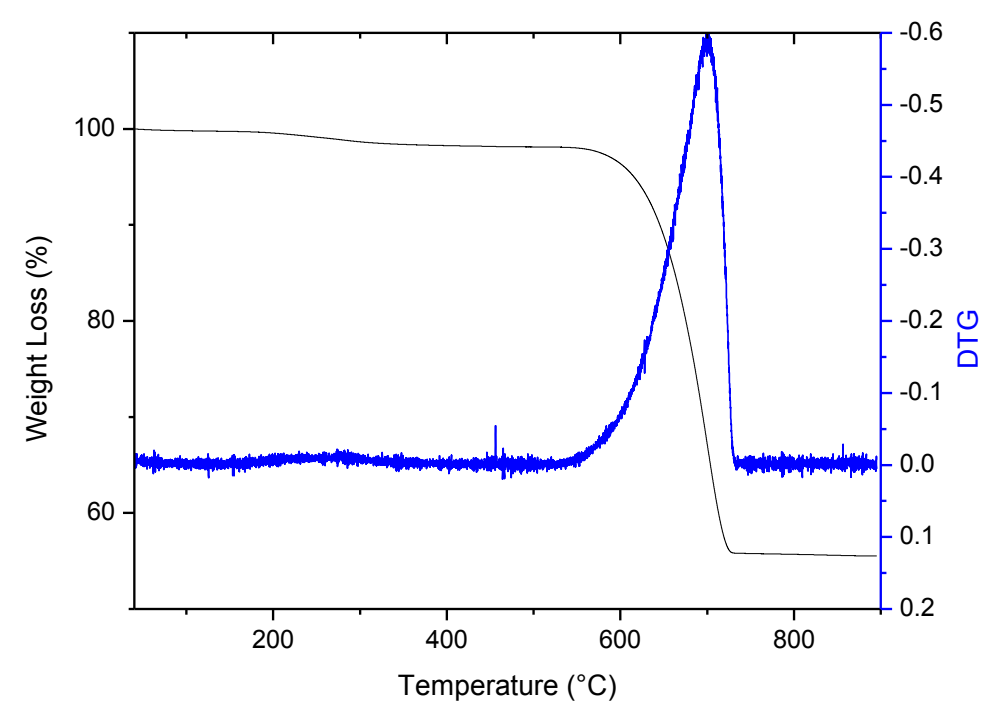

Figura 2: TG/DTG da ostra natural.

\subsection{Avaliação do Tempo de Equilíbrio}

O tempo de equilíbrio para a reação de transesterificação utilizando o resíduo da farinha da concha das ostras foi determinado processando a reação durante 6 horas. Os resultados (Figura 3) mostram que a conversão do óleo de girassol em ésteres metílicos aumenta à medida que aumenta o tempo reacional até 5 horas, e em seguida, tende a diminuir a partir de 6 horas. A conversão máxima foi obtida em 5 horas, correspondendo a igual a 96,58 wt. \%.

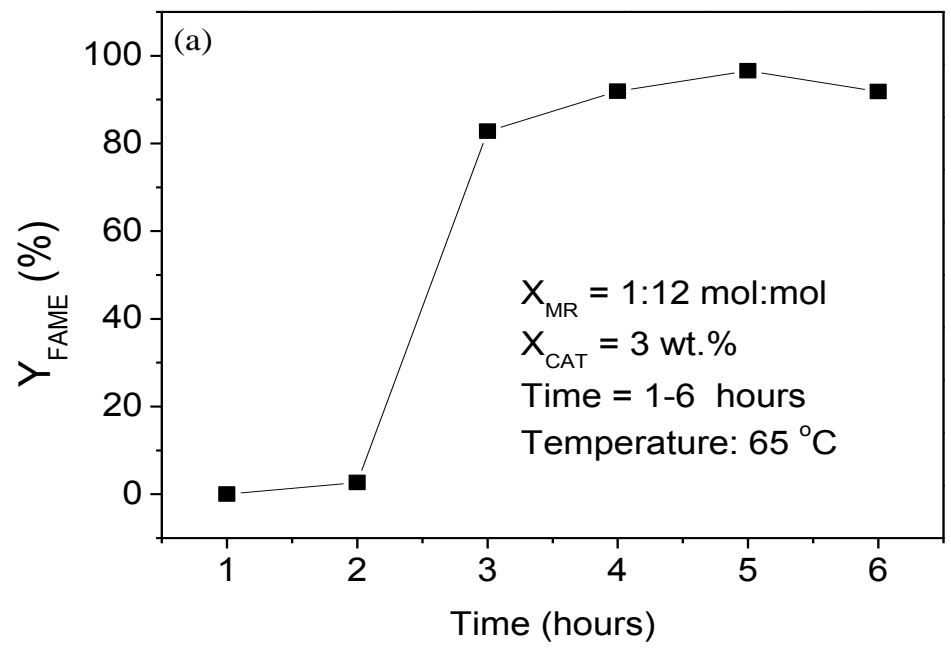

Figura 3: Efeito do tempo na reação de transesterificação para produção dos $Y_{\text {FAME. }}$

\subsection{Planejamento Fatorial}

O ajuste do modelo foi realizado por análise de variância (ANOVA), apresentada na Tabela 2. O valor de $\mathrm{F}$ calculado $(35,02)$ foi superior ao $\mathrm{F}$ tabelado $(4,77)$, no entanto, o modelo empírico 
proposto para a farinha da concha das ostras é válido com um nível de confiança de $95 \%$ estudado. A Equação (1) obtida é válida dentro do alcance experimental estudado.

$Y_{\text {FAME }}=99,01+0,47 X_{M R}-3,01 * X_{M R}^{2}+2,51 * X_{C A T}-8,01 X_{C A T}^{2}$

Tabela 2: ANOVA para o modelo empírico da farinha da concha das ostras.

\begin{tabular}{l|l|l|l|l}
\hline & Soma dos quadrados & Graus de liberdade & Quadrado médio & Fcal \\
\hline Regressão & 273,85223 & 4 & 68,46305 & 35,02 \\
Resíduos & 9,77317 & 5 & 1,954634 & \\
Total & 283,6254 & 9 & & \\
\hline
\end{tabular}

O diagrama de Pareto (Figura 4) mostra as variáveis significativas e insignificativas. A variável significativa sobre a resposta $\left(\mathrm{Y}_{\mathrm{FAME}}\right)$ foi $\mathrm{X}_{\text {CAT }}$ para um intervalo de confiança de $95 \%$, isto é, ao aumentar a $X_{C A T}$, consequentemente, aumento da $Y_{F A M E}$ é favorecido na reação de transesterificação do óleo de girassol com metanol.

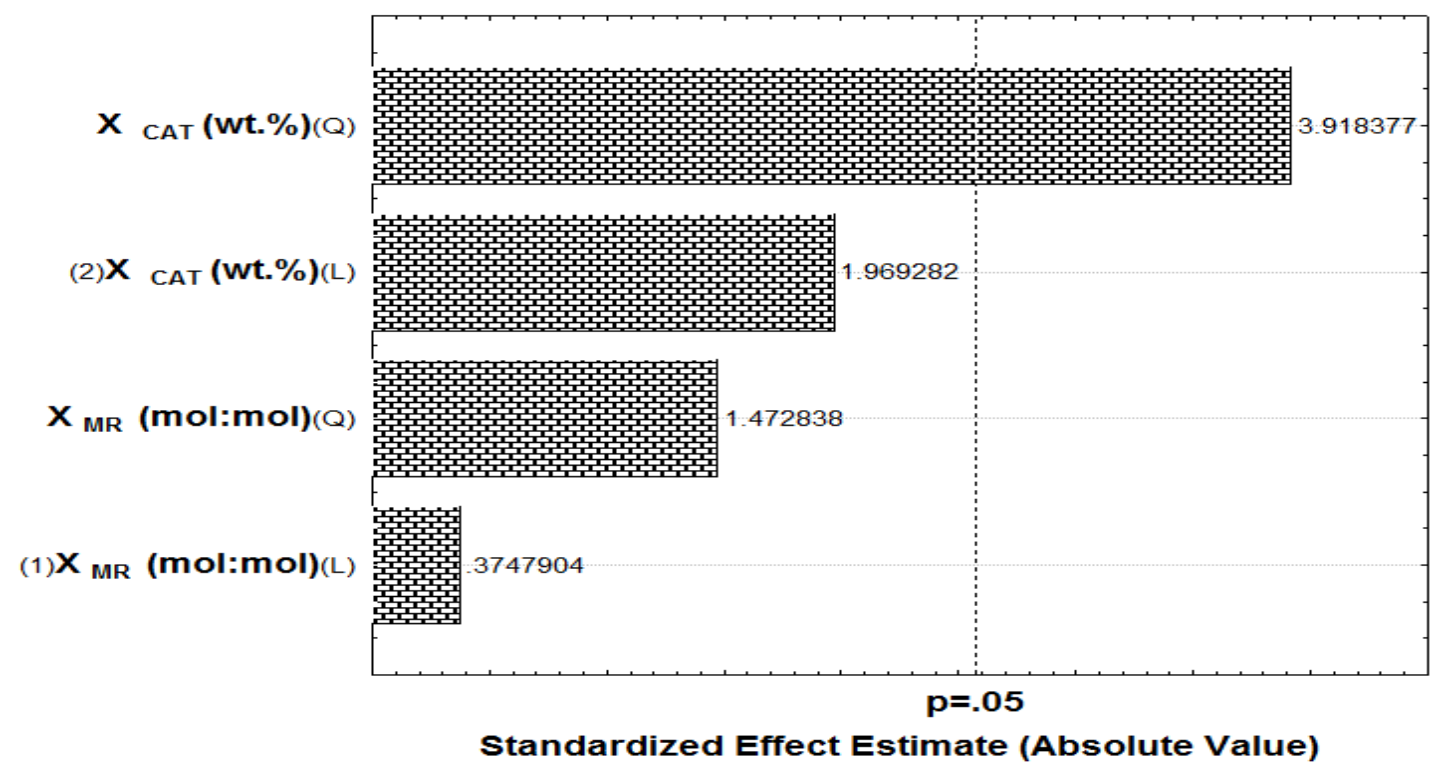

Figura 4: Gráfico de Pareto para o modelo.

A Figura 5 mostra o gráfico de superfície de resposta para farinha da concha das ostras, onde foi observado que a faixa estudada de $\mathrm{X}_{\mathrm{CAT}}$ (1-3 wt.\%) está relacionada com a conversão do produto final de $\mathrm{Y}_{\text {FAME }}$ (wt.\%). Entretanto para se obter elevadas conversões de $\mathrm{Y}_{\mathrm{FAME}}$, não é necessário utilizar na reação de transesterificação a $X_{\text {CAT }}$ máximo (3 wt.\%).

Uma análise importante para o planejamento fatorial realizado é a soma dos resíduos. De acordo com Montgomery (2001), a análise dos resíduos é imprescindível para avaliar o ajuste de qualquer modelo empírico. Um modelo que deixe muitos resíduos não é adequado. Em um modelo ideal não haveria resíduos, isto é, os resultados obtidos seriam semelhantes aos previstos. Para o catalisador heterogêneo da farinha da concha das ostras a soma do resíduo gerado foi de 9,77317 considerado um valor baixo, de modo que o modelo se classifica como próximo da idealidade. 


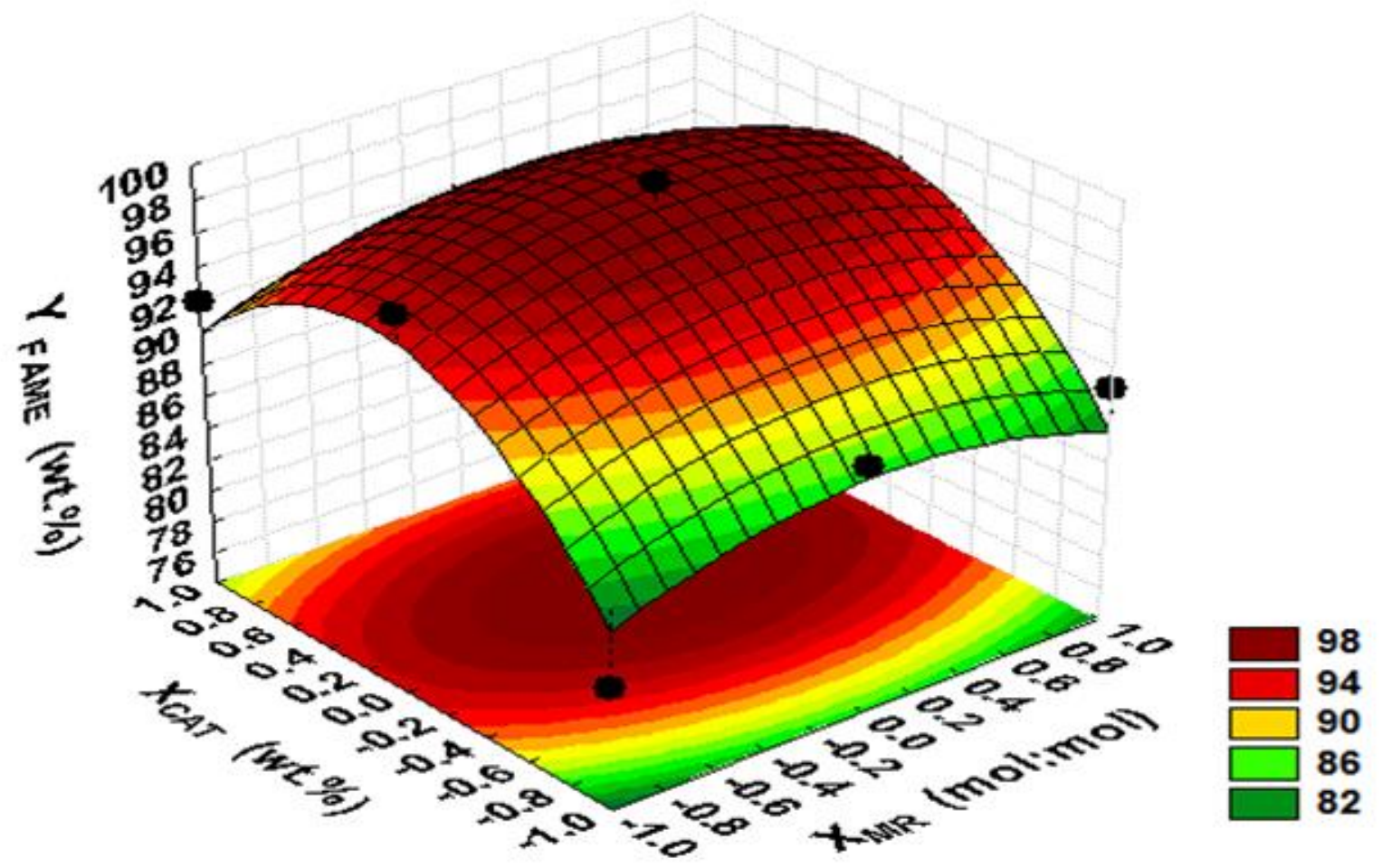

Figura 5: Superfície de resposta para o modelo.

A Y FAME máxima obtida foi de $99,99 \pm 0.04$ wt.\% de ésteres metílicos, obtida no ponto central do planejamento fatorial, nas seguintes condições reacionais: $X_{M R}$ de 1:09, $X_{C A T}$ de 2 wt.\%, temperatura de $65{ }^{\circ} \mathrm{C}$ e tempo de 5 horas conforme apresentado na Tabela 3 . Os resultados obtidos neste trabalho são compativeis com os alcançados com outros materiais derivados de fontes naturais para a produção de óxido de cálcio, como casca de ovo no trabalho de Correia et al. 2014) com o qual obteve-se uma conversão de $97,75 \%$ em ésteres metílicos. No trabalho de Buasri et al. (2014) com um catalisador obtido de conchas de ostras foi alcançado uma conversão de $93 \%$ em ésteres metílicos.

$\mathrm{O}$ aumento na $\mathrm{X}_{\mathrm{CAT}}$ implica no maior número de sítios básicos ativos no meio reacional, resultando em maiores conversões em ésteres metílicos. A variável $X_{\text {CAT }}$ tem seu processo de difusão nos reagentes, causando separação de fase e inibindo a reação de transesterificação. $\mathrm{Na}$ variável $X_{R M}$ o excesso de álcool desloca o equilíbrio químico da reação em direção à formação dos ésteres metílicos, aumentando a conversão de $\mathrm{Y}_{\text {FAME. }}$. Porém, o uso de um alto volume de álcool metílico dificulta o processo de separação por gravitação entre as fases do éster e glicerina formados, aumentando sua miscibilidade e favorecendo o deslocamento do equilíbrio no sentido inverso, ou seja, em direção à formação de mono-, di- e -triglicerídeos, diminuindo a produção dos $Y_{\text {FAME }}$. Deste modo verifica-se a importância da avaliação deste parâmetro através da análise via planejamento fatorial, de forma a mensurar a quantidade ideal de álcool a ser utilizado na transesterificação. 
Tabela 3 : Matriz do planejamento fatorial da reação de transesterificação com a ostra calcinada a $900{ }^{\circ} \mathrm{C} / 3 \mathrm{~h}$.

\begin{tabular}{|c|c|c|c|c|c|c|}
\hline \multirow{2}{*}{ Experimento } & \multicolumn{2}{|c|}{ Variáveis codificadas } & \multicolumn{3}{|c|}{ Variáveis reais } & \multirow{2}{*}{$\begin{array}{l}\text { Conversão } \\
Y_{\text {FAME }}(\% \text { p/p) }\end{array}$} \\
\hline & $\mathbf{X}_{\mathrm{RM}}$ & $X_{\text {CAT }}$ & $X_{R M}$ & $\mathbf{X}_{\mathrm{CAT}}$ & $\mathbf{X}_{\mathrm{TIME}}$ & \\
\hline 1 & -1 & -1 & $1: 6$ & 1 & 5 & $81,51 \pm 0,01$ \\
\hline 2 & 1 & -1 & $1: 6$ & 2 & 5 & $97,15 \pm 0,07$ \\
\hline 3 & -1 & 1 & $1: 6$ & 3 & 5 & $91,86 \pm 0,30$ \\
\hline 4 & 1 & 1 & $1: 9$ & 1 & 5 & $89,29 \pm 0,02$ \\
\hline 5 & 0 & 0 & $1: 9$ & 2 & 5 & $99,99 \pm 0,04$ \\
\hline 6 & 1 & 0 & $1: 9$ & 3 & 5 & $90,76 \pm 0,13$ \\
\hline 7 & -1 & 0 & $1: 12$ & 1 & 5 & $88,61 \pm 0,02$ \\
\hline 8 & 1 & 0 & $1: 12$ & 2 & 5 & $92,91 \pm 0,02$ \\
\hline 9 & 0 & -1 & $1: 12$ & 3 & 5 & $91,87 \pm 0,20$ \\
\hline 10 & 0 & 0 & $1: 9$ & 2 & 5 & $99,97 \pm 0,05$ \\
\hline 11 & 0 & 0 & $1: 9$ & 2 & 5 & $99,95 \pm 0,01$ \\
\hline 12 & 0 & 0 & $1: 9$ & 2 & 5 & $99,91 \pm 0,02$ \\
\hline
\end{tabular}

\section{CONCLUSÃO}

Os resultados de conversão observados neste trabalho $(99,99 \%)$ comprovam a viabilidade da produção de biodiesel utilizando catalisador obtido a partir da farinha de conchas de ostras.

Com o planejamento Plackett Burman, foi possível identificar as variáveis razão álcool:óleo e quantidade de catalisador como as de efeito mais significativo. Da analise do planejamento fatorial foi obtida uma equação polinomial de segunda ordem no intuito de avaliar o processo. O rendimento mássico ao nível de $95 \%$ de confiança apresentou um valor estatisticamente significativo, de acordo com a análise de variância, encontrando um modelo ajustado para explicar o processo reacional.

O planejamento estabeleceu como condições reacionais ótimas: razão óleo:metanol de 1:09 e quantidade de catalisador de $2 \%$. Nessas condições experimentais rendimento máximo calculado foi de $99,99 \%$ ( \pm 0,04 wt) em ésteres metílicos.

A partir dos resultados alcançados pode-se concluir que o planejamento fatorial é uma excelente ferramenta para avaliação de novos catalisadores para o processo de produção do biodiesel. 


\section{REFERÊNCIAS}

Alcântara, R., Amores, J., Canoira, L. T., Fidalgo, E., Franco, M. J., \& Navarro, A. (2000). Catalytic production of biodiesel from soy-bean oil, used frying oil and tallow. Biomass and bioenergy, 18(6), 515-527.

Al-Widyan, M. I., \& Al-Shyoukh, A. O. (2002). Experimental evaluation of the transesterification of waste palm oil into biodiesel. Bioresource technology, 85(3), 253-256.

Antolın, G., Tinaut, F. V., Briceno, Y., Castano, V., Perez, C., \& Ramırez, A. I. (2002). Optimisation of biodiesel production by sunflower oil transesterification. Bioresource technology, 83(2), 111-114.

Buasri, A., Rattanapan, T., Boonrin, C., Wechayan, C., \& Loryuenyong, V. (2015). Oyster and Pyramidella shells as heterogeneous catalysts for the microwave-assisted biodiesel production from Jatropha curcas oil. Journal of Chemistry, 2015, 1-7.

Correia, L. M., Saboya, R. M. A., de Sousa Campelo, N., Cecilia, J. A., Rodríguez-Castellón, E., Cavalcante, C. L., \& Vieira, R. S. (2014). Characterization of calcium oxide catalysts from natural sources and their application in the transesterification of sunflower oil. Bioresource technology, 151, 207-213.

Demirbas, A. (2009). Progress and recent trends in biodiesel fuels. Energy conversion and management, 50(1), 14-34.

Domingos, A. K., Saad, E. B., Wilhelm, H. M., \& Ramos, L. P. (2008). Optimization of the ethanolysis of Raphanus sativus (L. Var.) crude oil applying the response surface methodology. Bioresource Technology, 99(6), 1837-1845.

Gomes, J. F., Puna, J. F., Bordado, J. C., \& Correia, M. J. N. (2008). Development of heterogeneous catalysts for transesterification of triglycerides. Reaction Kinetics and Catalysis Letters, 95(2), 273-279.

Lôbo, I. P., Ferreira, S. L. C., \& Cruz, R. S. D. (2009). Biodiesel: parâmetros de qualidade e métodos analíticos. Química Nova, 32, 1596-1608.

Meher, L. C., Sagar, D. V., \& Naik, S. N. (2006). Technical aspects of biodiesel production by transesterification-a review. Renewable and sustainable energy reviews, 10(3), 248-268.

Montgomery, D. C. (2001). Design and analysis of experiments. John Wiley and sons.

Oliveira, J. F. G., Lucena, I. L., Saboya, R. M. A., Rodrigues, M. L., Torres, A. E. B., Fernandes, F. A. N., ... \& Parente, E. J. S. (2010). Biodiesel production from waste coconut oil by esterification with ethanol: The effect of water removal by adsorption. Renewable Energy, 35(11), 25812584.

Pinto, A. C., Guarieiro, L. L., Rezende, M. J., Ribeiro, N. M., Torres, E. A., Lopes, W. A., ... \& Andrade, J. B. D. (2005). Biodiesel: an overview. Journal of the Brazilian Chemical Society, 16(6B), 1313-1330.

Silva, D. (2007). Resíduos sólidos da malacocultura: caracterização e potencialidade de utilização de conchas de ostras (Crassostrea gigas) e mexilhão (Perna perna) (Dissertação de Mestrado). Universidade Federal de Santa Catarina, Florianópolis. 